\title{
Asymmetric properties between the forward and backward stimulated emission generated by ultrafast three- and four-photon excitation
}

\author{
Guang S. He, ${ }^{*}$ Changgui Lu, Qingdong Zheng, Alexander Baev, Marek Samoc, and Paras N. Prasad \\ The Institute for Lasers, Photonics and Biophotonics, State University of New York at Buffalo, Buffalo, New York 14260, USA
}

(Received 31 August 2005; revised manuscript received 31 October 2005; published 22 March 2006)

\begin{abstract}
This paper presents the observation of asymmetric behavior between the forward and backward stimulated emission, generated in multiphoton active dye solutions, through three- or four-photon excitation of subpicosecond laser pulses. At a pump energy level considerably higher than the lasing threshold value, the peak wavelengths of the forward stimulated emission are 20-30-nm shorter than those of the backward stimulated emission for the two investigated stilbazolium dye solutions (PRL-L3 and PRL-L10). This obvious spectral asymmetry can be explained by the following three considerations: (i) the difference of spatial/temporal sequences between the forward and backward stimulated emission pulses; (ii) blueshift of the peak wavelength of transient gain experienced by the forward stimulated emission pulse; and (iii) saturation of reabsorption at the forward lasing wavelength range. These proposed explanations are verified by a specially designed pumpprobe experiment, utilizing a white-light continuum as the probe beam and the $\sim 1300$-nm laser radiation as the pump beam for three-photon excitation. The experimental results have clearly shown the existence of the saturation effect of reabsorption and the gain-peak blueshift effect as well as their transient features.
\end{abstract}

DOI: 10.1103/PhysRevA.73.033815

PACS number(s): 42.65.Re, 42.50.Hz, 78.45.+h

\section{INTRODUCTION}

Since the middle of 1990 s, the two-photon pumped (2PP) frequency-upconversion lasing in dye solutions and dyedoped polymer matrices has become one of the most interesting subjects in nonlinear optics and quantum electronics [1-9]. As the pump mechanism in these cases is based on two-photon absorption which is a third-order nonlinear $\left(\chi^{(3)}\right)$ process, a pulsed laser source with high peak power is needed. In these cases cavity lasing was observed, and the output beams leaving the cavity in the forward and backward directions have the same spectral features. In 2002, the first observation of three-photon pumped (3PP) stimulated emission (cavityless lasing) in (APSS) dye solution was reported, which also indicated the same output wavelength for both the forward and backward stimulated emission pulses [10]. Furthermore, in 2003, a similar 3PP stimulated emission action was achieved in another (ASPI) dye solution; however, there was a considerable wavelength difference $(\sim 10 \mathrm{~nm})$ between the forward and the backward stimulated emission pulses [11]. At that time, the reported spectral asymmetry was unexplainable. In the case of 3PP stimulated emission, the pump mechanism is based on three-photon absorption which is a fifth-order nonlinear $\left(\chi^{(5)}\right)$ process; therefore an ultrashort laser pulse source is required to provide a much higher peak power [12].

More recently, we have extended our effort to achieve four-photon pumped (4PP) stimulated emission in solutions of a number of newly synthesized dyes [13]. In this particular case, the population inversion is based on direct fourphoton absorption that is a seventh-order nonlinear $\left(\chi^{(7)}\right)$ process; therefore, ultrashort laser pulse sources with much higher power levels and much longer wavelengths are

*Electronic address: gshe@acsu.buffalo.edu needed. The three- and four-photon pumped stimulated emission properties reported in the present work are based on two selected stilbazolium dyes synthesized in our research group. In comparison with other tested dyes [13], these two compounds exhibit a lower pump energy threshold and higher efficiency for stimulated emission under the 3PP or 4PP conditions. Most interestingly, it is found that at high pump levels there is a remarkable wavelength difference $(20-30 \mathrm{~nm})$ between the forward stimulated emission beam and the backward stimulated emission beam. In this paper we propose possible mechanisms leading to the observed asymmetric behavior between these two stimulated emission output beams from the same lasing medium. The validity of the proposed explanations is established by a specially designed two-beam (pump-probe) and time-resolved experiment.

\section{MULTIPHOTON ACTIVE LASING DYES}

The molecular structures of the two stilbazolium dyes (PRL-L3 and PRL-L10) are shown in the top-right corner of Fig. 1(a). The linear absorption spectra of the solutions of these two dyes in dimethylsulfoxide (DMSO) at a low concentration level $(0.0002 M)$ are shown in Fig. 1(a), while the linear transmission spectra of these two dye solutions at a high concentration level $(0.02 M)$ are given in Fig. 1(b). From Fig. 1(a) we can see that the linear absorption peaks for these two dye solutions are located at $\sim 452 \mathrm{~nm}$ for PRL-L3 and $\sim 430 \mathrm{~nm}$ for PRL-L10, respectively. From Fig. 1(b) it can be seen that for $1-\mathrm{cm}$ path length and $0.02 M$ concentration solutions used for lasing operation, the wavelength position on the red edge of the dye absorption band with $50 \%$ transmission is located at $587 \mathrm{~nm}$ for PRL-L3 and $550 \mathrm{~nm}$ for PRL-L10. For $70 \%$ transmission, the corresponding wavelength position is located at $601 \mathrm{~nm}$ for PRL-L3 and $560 \mathrm{~nm}$ for PRL-L10, respectively. For each of the measured 

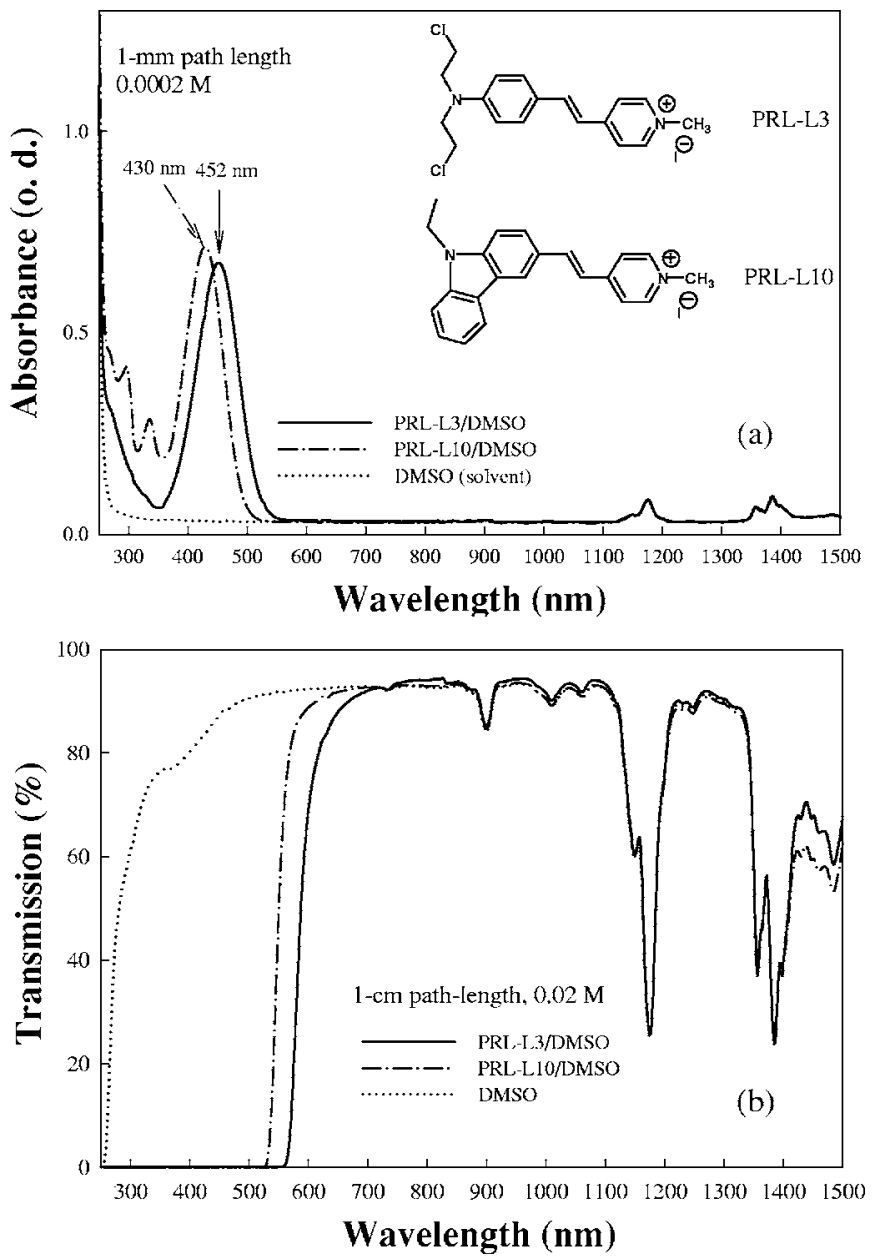

FIG. 1. (a) Absorption spectra of two dye solutions at low concentration levels, the molecular structures of the two dyes are shown in the top-right corner; (b) transmission spectra of two dye solutions at high concentration levels.

transmission curves in Fig. 1(b), $~ 8 \%$ loss is due to the reflection by the two optical windows of the solution cell. For a high concentration sample of 1-cm length, the residual linear absorption at the red edge of the absorption band in the visible wavelength range will exert an essential influence on the peak stimulated emission wavelength position, which is an important factor as we shall discuss in Sec. V.

Three-photon pumped (3PP) stimulated emission can be readily observed in these two dye solutions in DMSO, with $1-\mathrm{cm}$ pass length and $0.02 M$ concentration. In this case, the pump beam was the $\sim 1.3-\mu \mathrm{m}$ output from an optical parametric generator (OPG) pumped by $\sim 775$-nm and $\sim 160$-fs laser pulses from a Ti-sapphire oscillator/amplifier system (CPA-2010 from Clark-MXR). The reasons for choosing DMSO as the solvent are (i) a high solubility for the lasing dyes in it and (ii) a good transmission window around $1.3 \mu \mathrm{m}$, as shown in Fig. 1(b).

Similarly, we could also achieve four-photon pumped (4PP) stimulated emission in these two dye solutions in the deuterated solvent DMSO- $d_{6}$, with the same path length and concentration, but using the $\sim 1.9-\mu \mathrm{m}$ pump beam that was obtained from the same OPG system at a different tuning position. In this case, DMSO- $d_{6}$ is chosen as the solvent, because there is a good transmission window around 1.9-2.1 $\mu \mathrm{m}$.

The pump pulses at $\sim 1.3 \mu \mathrm{m}$ (for three-photon pumping) and $\sim 1.9-\mu \mathrm{m}$ (for four-photon pumping) wavelengths had a repetition rate of $1 \mathrm{kHz}$ and a divergence angle of $\leqslant 0.5 \mathrm{mrad}$. The pump beam was focused through a $f=10 \mathrm{~cm}$ lens onto the center of a 1-cm-long fused silica cuvette filled with the dye solution of $0.02 M$ concentration. To eliminate the influence of reflections from the optical windows of the cuvette, the incident angle of the pump beam was kept at $\geqslant 5^{\circ}$. It is found that once the input pump pulse energy is higher than a certain threshold value, a highly directional and single-pass stimulated emission can be observed in both the forward and the backward directions with respect to the pump beam direction. The measured pump energy threshold of 3PP stimulated emission was $\sim 0.88 \mu \mathrm{J}$ for PRL-L3 and $\sim 1.2 \mu \mathrm{J}$ for PRL-L10 in DMSO. The measured threshold of 4PP stimulated emission was $\sim 2 \mu \mathrm{J}$ for PRL-L3 and $\sim 3.9 \mu \mathrm{J}$ for PRL-L10 in DMSO- $d_{6}$. The nearfield and far-field measurements showed that the forward and the backward stimulated emission beams basically had the same spatial structures as the input pump beam [13].

\section{SPECTRAL ASYMMETRY OF FORWARD AND BACKWARD STIMULATED EMISSION}

Under three-photon pump condition of using $\sim 1.3-\mu \mathrm{m}$ laser pulses, it is found that when the pump energy level is slightly higher than the threshold value, the spectral structures for both the forward and the backward stimulated emission pulses are nearly the same. However, when the input pump energy levels are considerably higher than the threshold value, the peak wavelength of the forward stimulated emission is obviously blueshifted, while that for the backward stimulated emission remains unchanged. For the solution sample of PRL-L3 in DMSO, the measured spectral structures of three-photon absorption induced fluorescence as well as the forward and backward stimulated emission output at different pump energy levels are shown in Fig. 2. To help determine the precise pump wavelength and the spectral linewidth, the spectrum of the second-harmonic generation (SHG) signal from the pump beam is also given in this figure as a dotted-line curve with a spectral resolution of $\sim 1 \mathrm{~nm}$. From Fig. 2 one can see three salient features: (i) the spectral width of stimulated emission is remarkably less than the fluorescence but greater than the pump emission; (ii) the spectral shape and peak wavelength position for the backward emission remain unchanged at different pump levels; and (iii) the spectral structure and peak wavelength position for the forward stimulated emission are dependent on pump levels. At high pump levels, the peak wavelength of the forward stimulated emission is blueshifted by $\sim 20 \mathrm{~nm}$ for the PRL-L3 solution sample. The similar spectral measurement results for the PRL-L10 solution in DMSO are also obtained, from which we see the same three features as mentioned above. The only difference is that for the sample of PRLL10, the peak wavelength shift of the forward stimulated emission at high pump levels is even greater, i.e., up to $\sim 30 \mathrm{~nm}$ (from 594 to $564 \mathrm{~nm}$ ). 


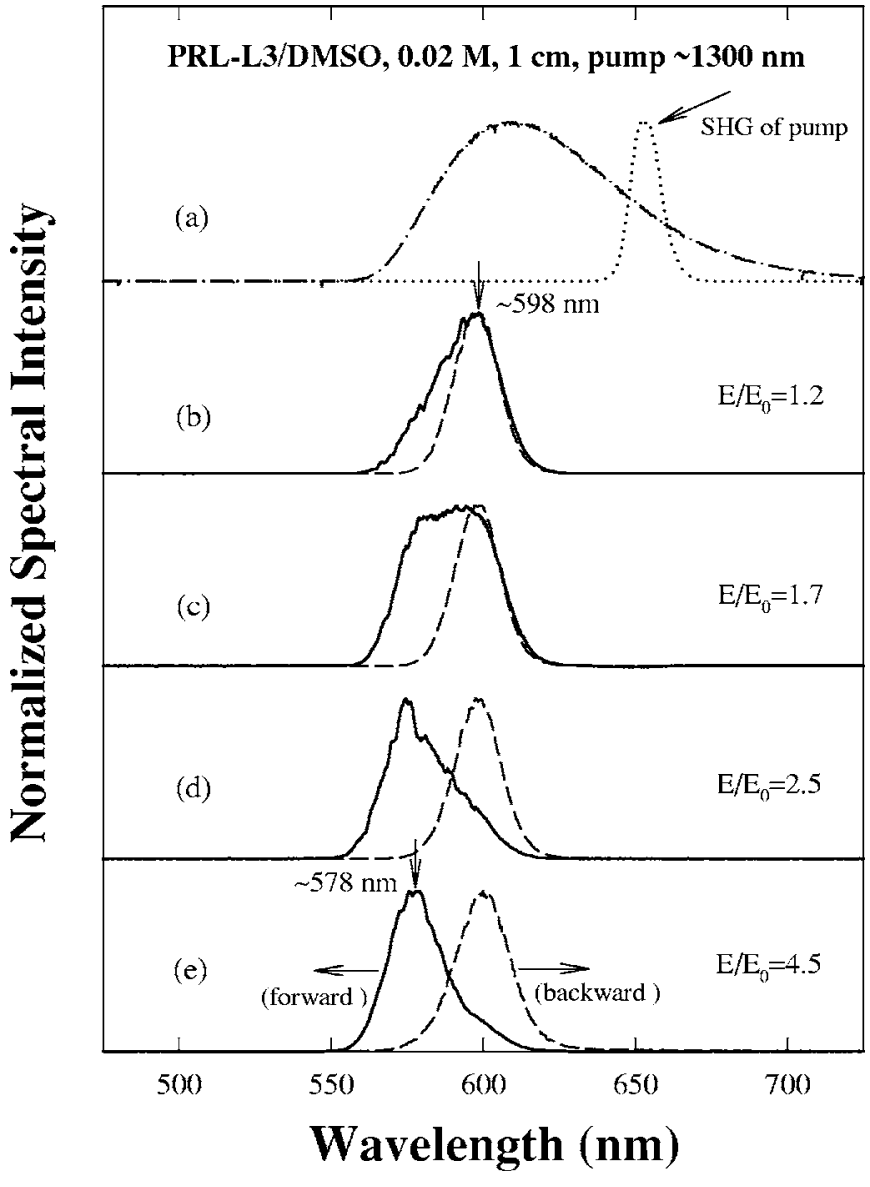

FIG. 2. Spectra of the three-photon induced fluorescence (a) and the forward (solid-line) and backward (dashed-line) stimulated emission (b)-(e) in the PRL-L3/DMSO solution at different pump levels. $E$ is the pump energy and $E_{0}$ is the threshold pump energy. The dotted-line curve is the spectrum of the SHG from the pump beam.

Similar spectral measurements are also performed with the same two dye compounds in DMSO- $d_{6}$ under fourphoton pump condition using $\sim 1$.9- $\mu$ m laser pulses. As an example, Fig. 3 shows the measured spectral structures of the four-photon absorption induced fluorescence emission as well as the stimulated emission at three different pump energy levels in the PRL-L3/DMSO- $d_{6}$ sample. One can see that the basic features shown in Fig. 3 under 4PP conditions are essentially the same as that shown in Fig. 2 under $3 \mathrm{PP}$ conditions.

\section{DYNAMIC SPECTRAL STRUCTURES OF FORWARD STIMULATED EMISSION}

All spectral data shown from Figs. 2 and 3 are timeintegrated results averaged over a great number of stimulated emission pulses (mostly over 1000 pulses for $1 \mathrm{~s}$ exposure). In order to know the temporal behavior of different spectral components of the stimulated emission output, a high-speed streak camera (C5690-22 from Hamamatsu) with 2-ps resolution was utilized to record the time-resolved spectral structure of a single stimulated emission pulse. To do so, the

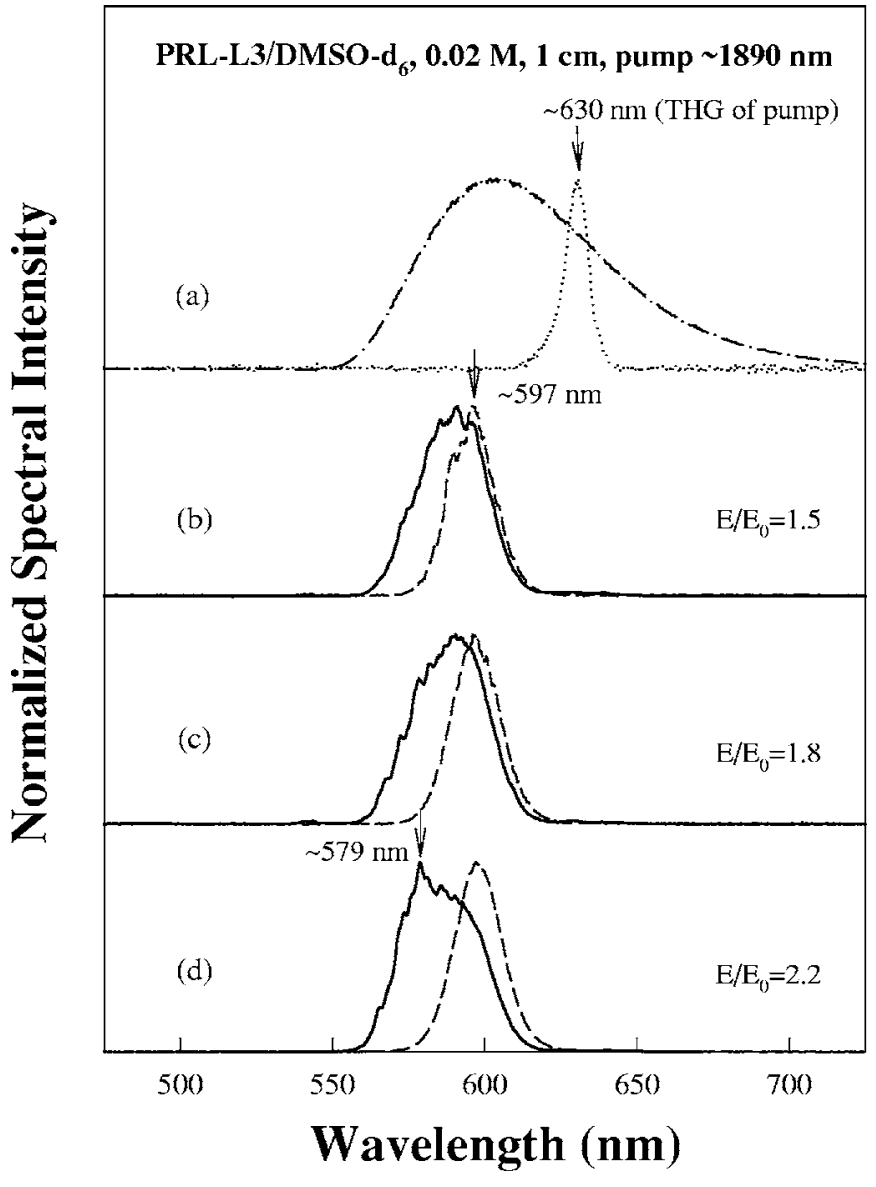

FIG. 3. Spectra of the four-photon induced fluorescence (a) and the forward (solid-line) and backward (dashed-line) stimulated emission (b)-(d) in the PRL-L3/DMSO- $d_{6}$ solution. The dotted-line curve is the spectrum of the third-harmonic generation (THG) from the pump beam.

collimated forward stimulated emission beam was first reflected by a dispersion grating of 600 groove $/ \mathrm{mm}$, and then was focused on the horizontal entrance slit of the streak camera. For the PRL-L3/DMSO solution under 3PP condition at three different pump energy levels, the measured dynamic behavior of three specifically chosen spectral components associated with the same single 3PP forward stimulated emission pulse is shown in Fig. 4. Among these three chosen wavelengths, the $\sim 600$-nm component is close to the peak stimulated emission wavelength position at a lower pump level [see Fig. 2(b)], the $\sim 590$-nm component is close to the central position at a medium pump level [see Fig. 2(c)], while the $\sim 580$-nm component is close to the stimulated emission peak at a high pump level [see Fig. 3(e)]. From Fig. 4 we can see the following three features: (i) at a low pump level, the forward stimulated emission occurs mainly around the 600-nm spectral range with a longer duration ( $\sim 50 \mathrm{ps})$; (ii) at a medium pump level, the forward stimulated emission occurs mainly around the 590-nm range with a much shorter duration (13-15 ps); and (iii) at a high pump level, the forward stimulated emission mainly occurs around the 580-nm range with a short duration (15-20 ps). These features are consistent with those shown in Fig. 2, but providing addi- 

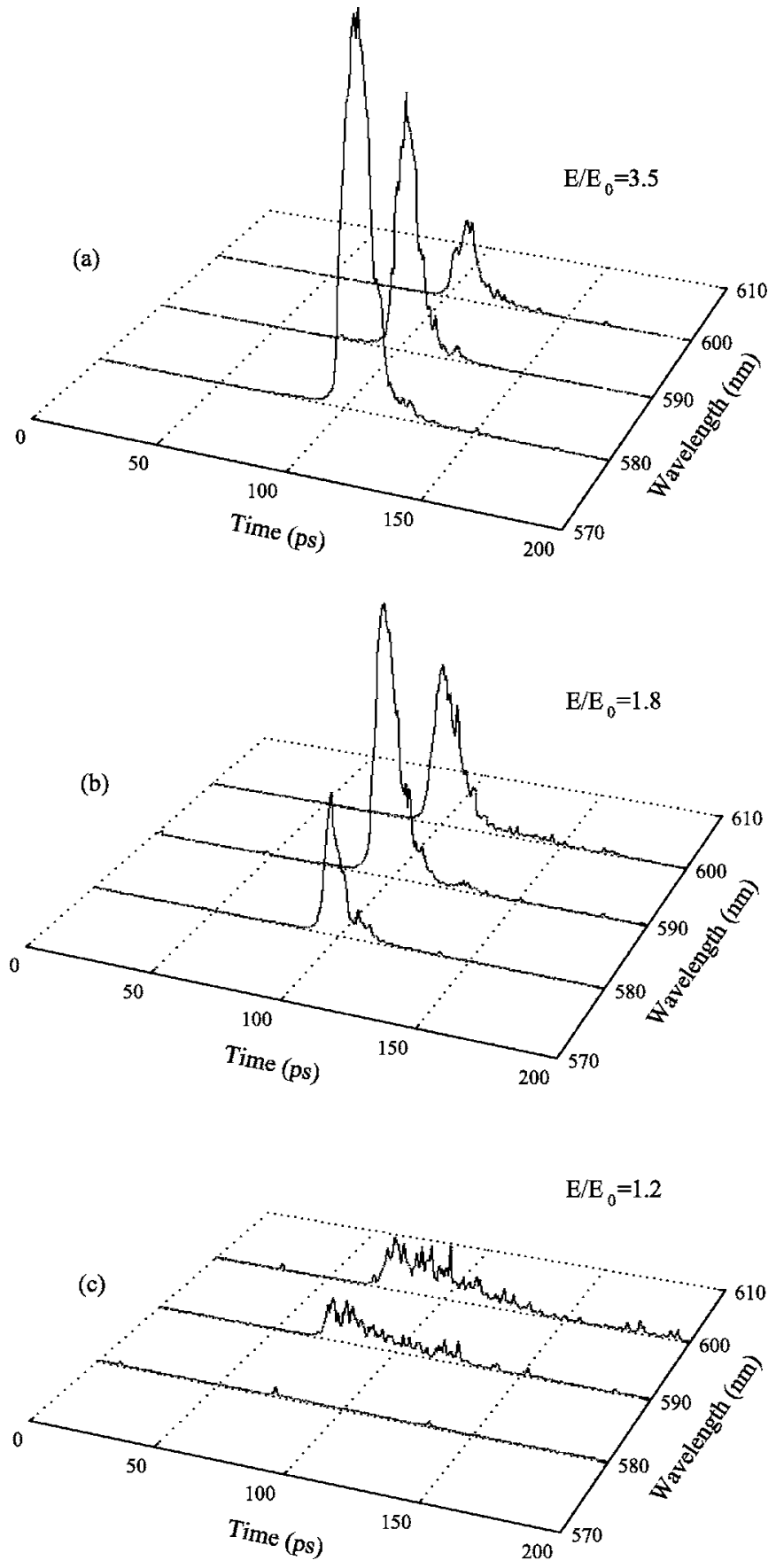

FIG. 4. Temporal profiles of three forward stimulated emission spectral components from the PRL-L3/DMSO solution under threephoton excitation conditions at different pump energy levels.

tional information of pulse duration of different spectral components. For the other (PRL-L10) dye sample, quite similar results were obtained under the same 3PP conditions. Furthermore, under 4PP conditions for both samples, we could see basically the same dynamic features of the forward stimulated emission as described above.

\section{PHYSICAL EXPLANATIONS OF THE OBSERVED EFFECTS}

Based on the experimental results described in the preceding two sections, we know the most important fact is that

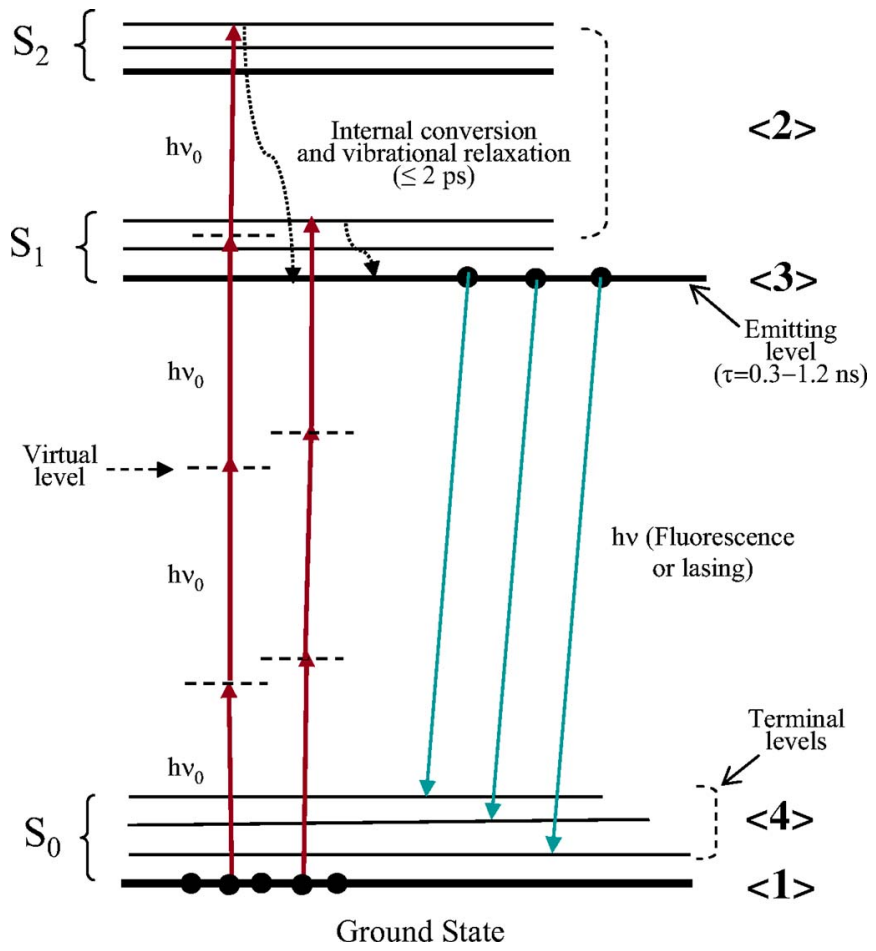

FIG. 5. (Color online) Simplified four-level model of multiphoton pumped lasing excitation processes.

under 3PP or 4PP conditions, when the pump intensity level increases, the peak wavelength position for the backward stimulated emission remains the same while the peak wavelength for the forward stimulated emission is blueshifted. To explain this apparently surprising behavior of the forward stimulated emission, we should consider the following three issues separately: (i) the difference of temporal sequence between forward and backward lasing pulses; (ii) the difference of gain maximum wavelength for forward and backward lasing pulses; and (iii) the difference of self-absorption influence on these two pulses.

First, we assume that a simplified four-level lasing model, shown in Fig. 5, is applicable to our case. According to such a model [14], at room temperature and without optical excitation, most dye molecules stay in the lowest vibrational state of ground electronic state $S_{0}$. By means of simultaneous three- or four-photon absorption, part of ground-state dye molecules can be excited to higher vibrational levels of a higher singlet electronic state $S_{i}(\mathrm{i}=1,2, \ldots$,$) . Those excited$ molecules will rapidly relax to the lowest vibrational level of $S_{1}$ through internal conversion and vibrational relaxation processes. Our experimental result described later shows that these processes take a time period of $\leqslant 2$ ps. Once the excited dye molecules reach the lowest vibrational level of $S_{1}$, which usually exhibits a longer lifetime of $10^{-8}-10^{-9} \mathrm{~s}$, the visible fluorescence emission occurs accompanying the transitions from this level to various vibrational levels of the ground state $S_{0}$. In our specific case, the measured 3PAinduced fluorescence lifetime is $\sim 680 \mathrm{ps}$ for PRL-L3/ DMSO and $\sim 1200$ ps for PRL-L10/DMSO, respectively. As mentioned before, at room temperature, the population in the upper vibrational levels of the ground state are negligible, 
Gain medium
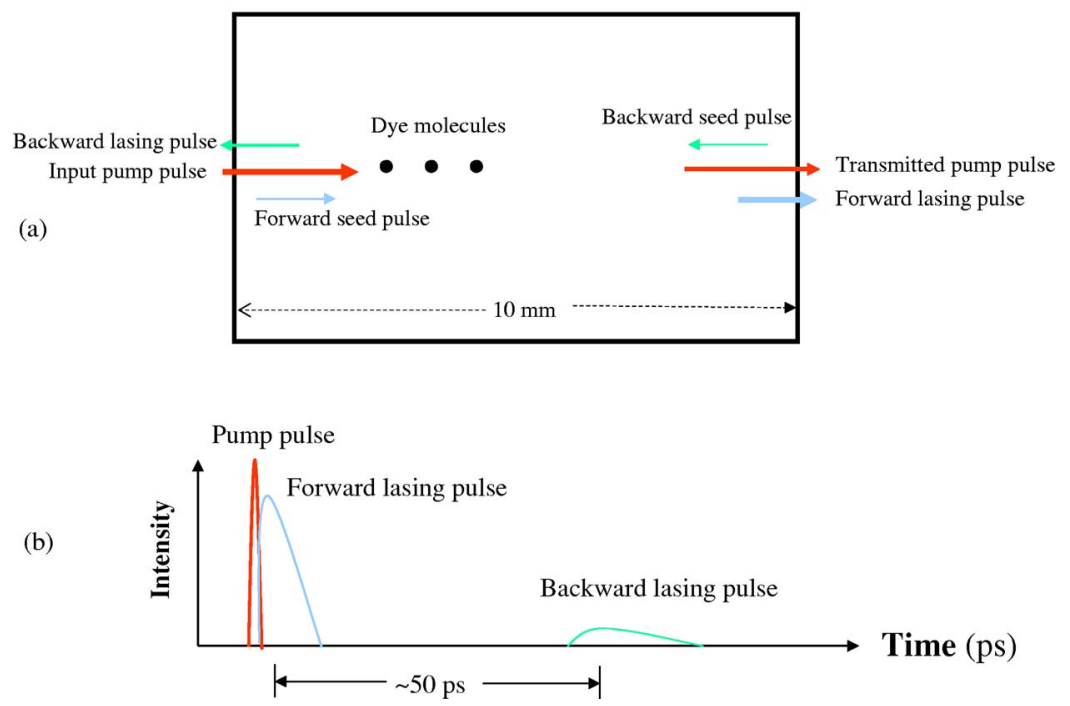

(b)
FIG. 6. (Color online) The spatial and temporal sequences of the forward and backward lasing pulses. thus the population inversion is relatively easy to build up. If the pump level is high enough that the stimulated gain based on the population inversion predominates over various loss mechanisms, the intense stimulated emission should be achieved.

\section{A. Temporal sequence of forward and backward lasing pulses}

To explore the physical origins that lead to different spectral behaviors between the forward and the backward stimulated emission pulses under our ultrafast multiphoton pump conditions, we shall first consider the differences of spacetime sequence between the forward and the backward stimulated emission pulses. For clarity, the schematic demonstrations of spatial and temporal sequences for these two pulses are shown in Figs. 6(a) and 6(b), respectively. In Fig. 6(a) we can image that the initial weak (seed) stimulated emission signal is formed following the input pump pulse with a $\leqslant 2$ ps delay determined by the internal conversion and vibrational relaxation processes. Furthermore, for a given group of dye molecules, for instance located in the position $\sim 1 / 3$ near to the front window of the dye cell, they will be excited by the pump pulse first and then provide a gain to the slightly delayed forward seed pulse of stimulated emission. On the contrary, in order to get the maximum gain length, the effective initial backward stimulated emission signal may start from the position near to the exit window of the dye cell, and then propagates toward the entrance direction. Hence, for the given excited dye molecules shown in Fig. 6(a), the backward lasing pulse arrives much later $(\sim 50 \mathrm{ps})$ than the forward lasing pulse. In our lasing experiment, the pump beam was focused by a $f=10$-cm lens on the center of the 1-cm sample cell, therefore the effective gain length providing the most amplification is estimated to be around the 4-5 $\mathrm{mm}$ range. Correspondingly, for those dye molecules shown in Fig. 6(a), the temporal sequences of the pump pulse, the forward and backward stimulated emission pulses are schematically shown in Fig. 6(b). Due to the competition of usage of the population inversion, the forward stimulated emission pulse will get much stronger amplification than the backward stimulated emission signal that arrives much later. This fact can be used to explain why under 3PP conditions, the measured forward lasing output energy was $\sim$ tenfold greater than the backward lasing output energy [13].

\section{B. Gain-peak wavelengths for forward and backward lasing pulses}

In a dye solution or dye-doped polymer rod, the fluorescence profile exhibits an inhomogeneous broadening feature, derived from various terminal vibrational states as well as distributed orientation interactions between the dye molecules and the solvent molecules. For a nanosecond-pulsed or cw-operation dye laser, the lasing peak wavelength is basically quite close to the corresponding fluorescence peak wavelength position within the inhomogeneously broadened profile, provided that there is no special spectral selection technique applied and the self-absorption at the fluorescence peak wavelength position can be neglected. In those cases, one can say that for a given lasing dye medium, the steadystate gain maximum is located at the same spectral position of the corresponding fluoresce maximum. However, in our experiment, the pump pulse is of subpicosecond duration, the forward stimulated emission pulse appears only 1-2 ps later than the pump pulse [13]. In this particular case, the transient gain behavior at early time for the forward lasing pulse might be essentially different from that experienced later by the backward lasing pulse. The forward lasing pulse would experience a transient gain peak located on the blue side of the fluorescence peak, which is provided by the excited dye molecules unrelaxed in the sense of dye-solvent interactions; whereas, when the solvent relaxation is accomplished, the later arrived backward lasing pulse experiences a steadystate gain, the peak of which is located in the center of the ordinary fluorescence spectral profile.

The dyes utilized for our lasing studies are stilbazolium salts with strong polarity, dissolved in polar solvents (such as 


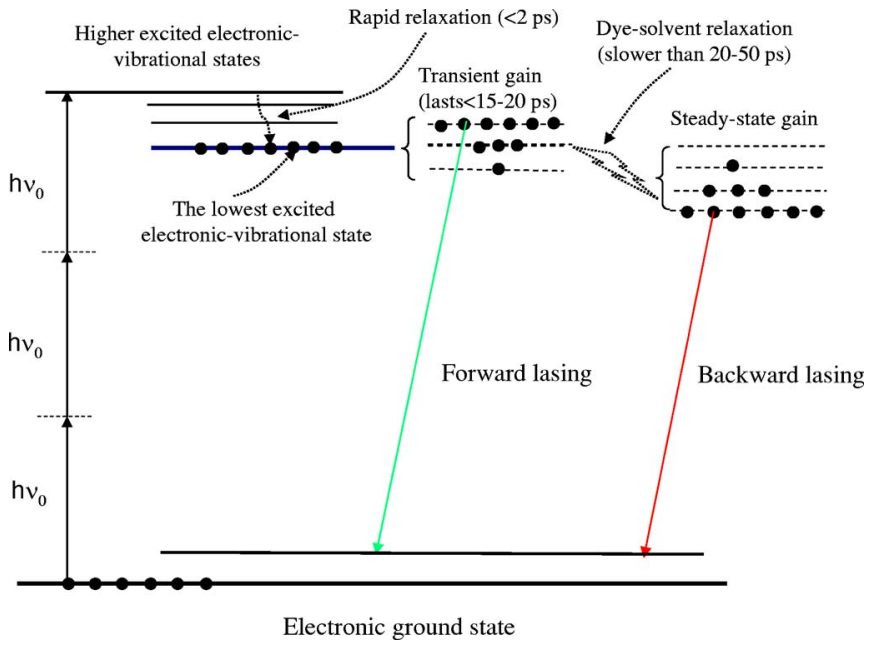

FIG. 7. (Color online) Detailed schematics of transient and steady-state gain responses with consideration of solvent relaxation.

DMSO or DMSO- $d_{6}$ ). One expects that, in this case, the interaction between a dye molecule and the surrounding solvent molecules should be quite strong, which may be the key to explain the peak wavelength difference between the transient gain and the steady-state gain. Without external excitation, this interaction leads to an equilibrium orientation distribution between the unexcited dye molecule and the surrounding solvent molecules. Upon excitation, however, there will be an instantaneous change of direction and/or magnitude of the electric-dipole moment of the excited dye molecules. Then local relaxation of the orientation between the excited molecules and the surrounding solvent molecules can take place, and after a certain period of time, a new equilibrium of orientation distribution will be established. This solvent relaxation may take several tens to $\sim 100 \mathrm{ps,}$ depending on the specific properties of dye and solvent [14]. It should be noted that during this relaxation process, reorientations between the excited dye molecules and the surrounding solvent molecules can take place only by exhausting part of internal energy of the excited dye molecules to overcome the reorientation viscosity. For this reason, the dye molecules belonging to the lowest level of $S_{1}$ will statistically have a higher energy height at the very beginning, then gradually lose part of their energy, and finally reach a steadystate orientation status with a lower energy height. Based on this consideration, the simplified four-level lasing model shown in Fig. 5 should be further modified as depicted in Fig. 7. The physical meaning implied by this new figure is that after a period of $\leqslant 2 \mathrm{ps,} \mathrm{most} \mathrm{of} \mathrm{the} \mathrm{excited} \mathrm{dye} \mathrm{mol-}$ ecules will relax to the lowest level of $S_{1}$, then there will be a transient molecular internal-energy distribution regarding the relative orientations between these dye molecules and their surrounding solvent molecules. After that, gradually the excited dye molecules and the surrounding solvent molecules have to readjust their relative orientations to reach a lower energy configuration [14]. Assuming that this relaxation takes a period of 30-50 ps in our specific case, the forward stimulated emission pulse will experience a gain with shorter peak wavelength, whereas the backward stimulated emission pulse will experience a gain with longer peak wavelength.

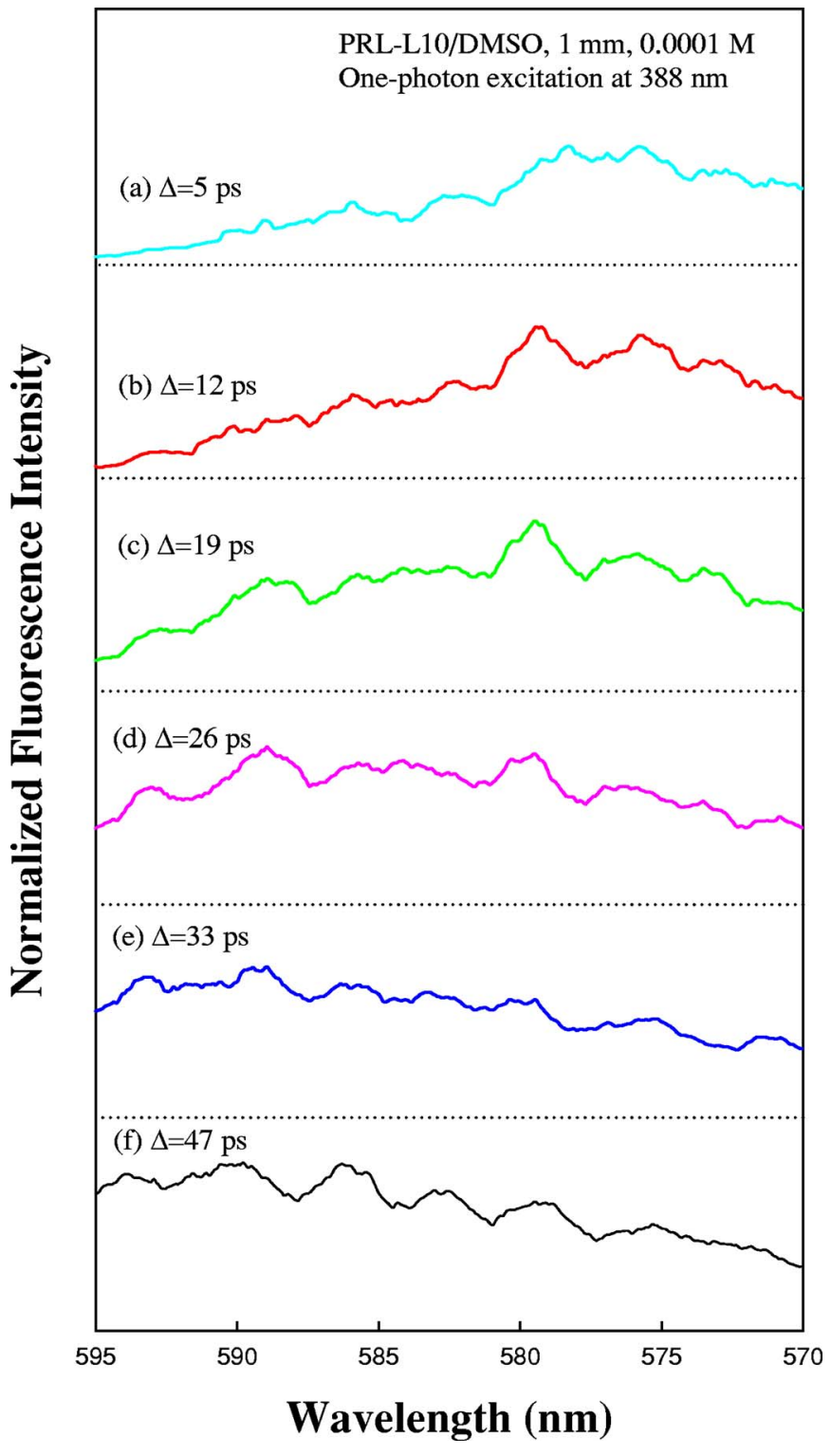

FIG. 8. (Color online) Transient fluorescence spectral profiles of the PLR-L10/DMSO solution measured at different time moments, excited by $388 \mathrm{~nm}$ and 160 fs laser pulses.

For a conventional fluorescence spectral measurement, the integrated time of measurement is much longer than the fluorescence lifetime, therefore, it is also much longer than the solvent-relaxation period. For this reason the measured steady-state fluorescence spectral profile will have a peak wavelength nearly the same as the gain maximum wavelength experienced by the backward lasing pulse. However, the transient fluorescence spectral profiles can also be obtained by using a high-speed streak camera in conjunction with a grating spectrometer. As an example, for a diluted PRL-L10/DMSO solution excited by one-photon absorption at $388 \mathrm{~nm}$, the transient fluorescence spectral profiles, sampled at different time delays from the starting moment of fluorescence emission, are shown in Fig. 8. From these measured spectral curves we can see that near to the beginning, the peak emission wavelength is around $575 \mathrm{~nm}$, and after $35-40 \mathrm{ps}$ it shifts to $\sim 590 \mathrm{~nm}$ position and then remains 


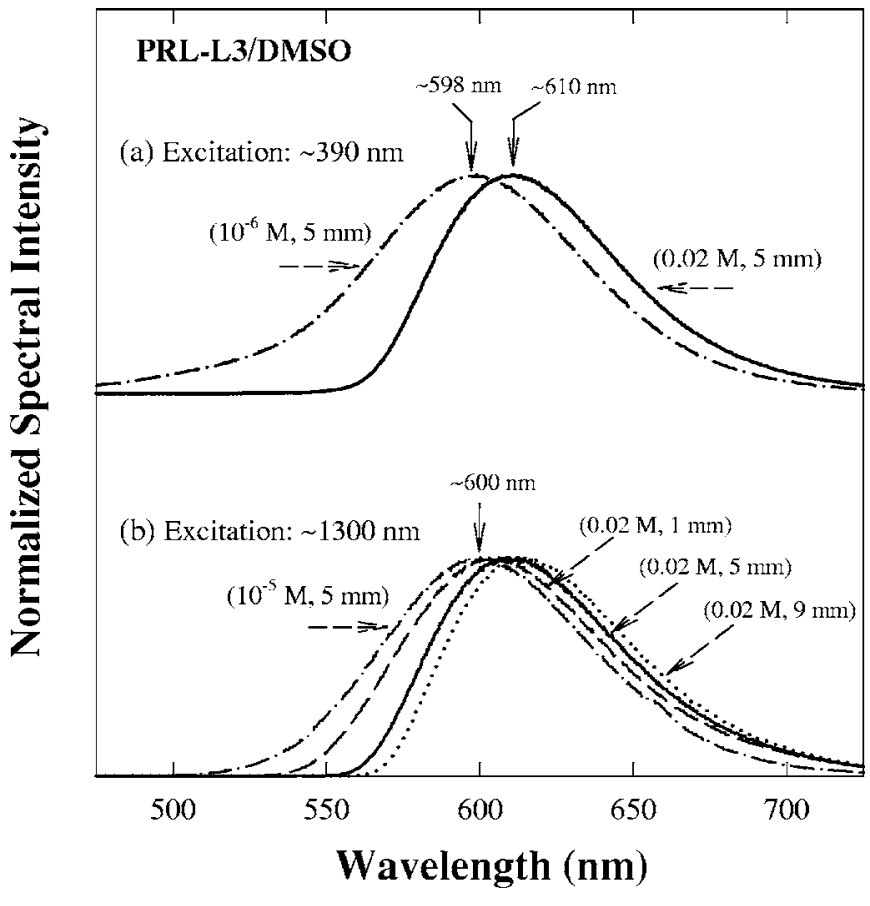

FIG. 9. Spectral curves of fluorescence emission in PRL-L3/ DMSO solutions with different concentration and propagationlength values upon (a) one-photon excitation and (b) three-photon excitation.

unchanged with the further delay. These results basically support our physical explanations.

\section{Transient saturation effect of self-absorption for the forward lasing pulse}

The statements presented in Sec. V B may explain the difference of gain peak wavelengths between the forward and backward lasing pulses. However, there is one more question that needs to be answered: Why does the difference between the forward and the backward lasing depend on the optical pump level? It is known that at a pump level slightly higher than the threshold, the forward lasing wavelength is quite close to the backward lasing wavelength. Only at a pump level much higher than the threshold, the forward lasing wavelength becomes much shorter than the backward lasing wavelength. To answer this question, we have to also consider the one-photon reabsorption of the visible emission with shorter wavelength as well as the saturation of this reabsorption at higher pump intensity.

To indicate the reabsorption influence on the apparent spectral profile of fluorescence emission, the measured onephoton excited fluorescence profiles of the PRL-L3/DMSO sample at two different concentration levels are shown in Fig. 9(a). In this case, the propagation lengths of the measured fluorescence signals inside the solution were kept the same $(\sim 5 \mathrm{~mm})$. One can see a considerable redshift of the apparent emission peak and the obvious cutoff of the blue wing of the emission band for the high concentration sample. This is due to re-absorption occurring in the range of $500-590 \mathrm{~nm}$, as shown by the corresponding transmission curve in Fig. 1(b) for the same concentration sample. Furthermore, a similar re-absorption influence can also be demonstrated in a high concentration sample of PRL-L3 solution excited by three-photon absorption. In this case, the spectral profiles corresponding to three different path lengths of measured fluorescence signals are shown in Fig. 9(b), indicating a greater redshift with an increased propagation distance in the high concentration solution.

It is known that the reabsorption effect is harmful for an original weak fluorescence signal that is going to be amplified through stimulated emission. In other words, a strong reabsorption in a given wavelength range may prohibit the effective formation of stimulated emission within this spectral range. From the spectral curves shown in Figs. 1(b) and 9(b), one can see that reabsorption at the $\sim 580 \mathrm{~nm}$ position is pretty strong for a $1-\mathrm{cm}$ long PRL-L3/DMSO solution of $0.02 M$ concentration; hence, no maximum lasing should occur at this wavelength position, even though the transient gain maximum may be close to this position for the forward pulse signal. This is true when the pump level is not considerably higher than the threshold. However, once the optical pump level is high enough and the ground-state dye molecules are mostly depopulated through multiphoton excitation, the re-absorption effect due to linear absorption from the ground-state dye molecules can be significantly reduced. In the latter case, the maximum forward lasing wavelength will be only determined by the transient gain maximum position, i.e., $580 \mathrm{~nm}$ for PRL-L3/DMSO sample. Similarly, we could also explain why at a high pump level the forward lasing peak wavelength for the PRL-L10/DMSO sample is located in the 564-574 $\mathrm{nm}$ range.

\section{VERIFYING EXPERIMENT AND RESULTS}

To verify the proposed explanations described above, we have pursued a time-resolved two-beam (pump-probe) experiment. The basic idea is to utilize a synchronized whitelight continuum generation pulse to detect the transient behavior of both the reabsorption bleaching effect and the gain peak blueshift effect, induced by strong ultrashort multiphoton excitation in a dye solution sample. The optical layout of this experiment is shown in Fig. 10. For the case of threephoton excitation, the incident pump of $\sim 1300 \mathrm{~nm}$ was first focused by an $f=5-\mathrm{cm}$ lens on a $2-\mathrm{mm}$ thick quartz plate to generate a white-light continuum beam. Then this continuum beam was separated from the pump beam by a $45^{\circ}$-placed beam splitter that reflected the former while transmitted the latter. After equal propagating distance, these two beams were focused through an $f=7-\mathrm{cm}$ lens onto the same focal spot near to the center of the lasing dye solution cuvette of 5-mm path length. The crossing angle between these two beams after the focusing lens was $\theta \approx 2.7^{\circ}$ (inside the solution). By adjusting the relative delay of the white-light probe beam, we could ensure temporal overlapping inside the gain medium between the strong monochromatic pump pulse and the relatively weak probe pulse of a specific spectral component within the range of the continuum spectrum. After passing through the gain medium cell, both the transmitted pump beam and the induced forward stimulated emission beam 


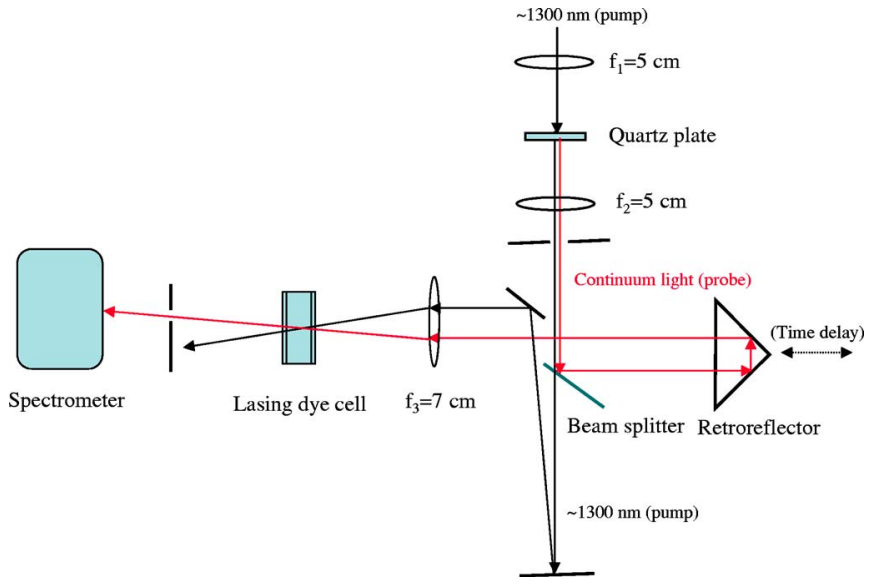

FIG. 10. (Color online) Optical setup for pump-probe and time resolved experiment. The pump beam of $\sim 1300 \mathrm{~nm}$ is for threephoton excitation, while the probe beam is a white-light continuum pulse for detecting transient behaviors of excited dye molecules.

were blocked by a screen, while the transmitted probe beam with a continuous spectral distribution could pass through a pinhole and then enter a grating spectrometer (HoloSpec from Kaiser Optical, Ann Arbor, Michigan). In such a way, the spectral intensity changes at different wavelength positions of the probe beam can be measured. Furthermore, the temporal behavior of such a spectral intensity change can also be studied by varying the relative delay between the pump pulse and the probe pulse.

The experimental results described below were obtained in the PRL-L3/DMSO solution sample of the 5-mm path length and $0.02 M$ concentration, pumped by $1300-\mathrm{nm}$ laser pulses at an energy level of approximately four times higher than the stimulated emission threshold value. The energy level of the probe pulse with white-light spectral distribution was attenuated to lower than $1 \mu \mathrm{J}$ to ensure that there was no bleaching effect or gain effect created by the probe pulse itself. Shown in Fig. 11 are the spectral intensity profiles of the transmitted probe pulse measured at different time-delay values between the pump pulse and the probe pulse. For comparison, the initial spectral profile of the incident probe pulse is given in Fig. 11(a) by a dotted-line curve, where the solid-line curve is the spectral profile of the transmitted probe pulse, measured when the probe pulse arrived 0.33 ps earlier than the pump pulse. In this case, the measured spectral-profile change indicates only the linear attenuation influence of the dye solution as a function of the wavelength. In Fig. 11(b), the curve was measured when the relative delay time was nearly zero between the pump pulse and the 580-nm component of the probe pulse. The curves shown in Figs. 11(c)-11(i) are obtained with different increasing delay-time values. Notice that the pump pulse will be temporally overlapping with different spectral components of the probe pulse at different delay times, owing to the groupvelocity dispersion (GVD) effect occurring in the dye solution. It means that the red spectral components of the probe pulse propagate faster than the blue components inside the dye solution.

From the curve shown in Fig. 11(b) we can see two interesting features: (i) there is an intensity hump around

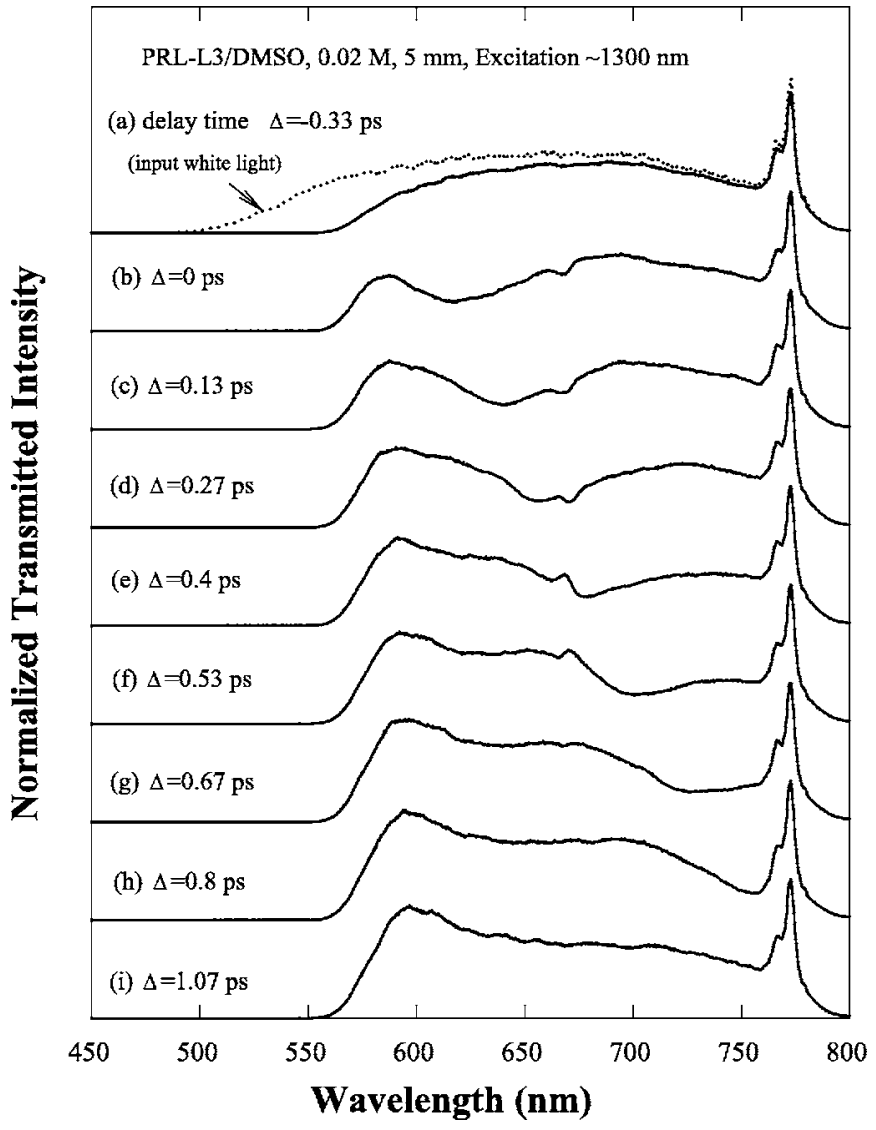

FIG. 11. Measured changes of spectral profiles of the transmitted probe pulse with different delay times. These changes indicate the bleaching effect of re-absorption, the excited-state absorption, as well as the blueshift effect of the gain peak in the PRL-L3/ DMSO solution.

580-590-nm position, which clearly shows the selfbleaching effect of the linear absorption in this range; (ii) there is an obvious dip around the 615-nm position, which is an additional attenuation induced by the excitedstate absorption (one-photon absorption from a given threephoton excited state to another higher state). From the curves shown in Figs. 11(b)-11(h) we can further see that the excited-state-absorption induced spectral dip shifts from $\sim 615$ - to $\sim 760$-nm position, within a delay change less than 1 ps. This apparent dip shift can be understood by considering the GVD effect as we mentioned before. When the time delay is greater than $1 \mathrm{ps}$, as shown in Fig. 11(i), the spectral dip can no longer be seen, which implies that the observed excited-state absorption took place only from those higher excited states than the lowest $S_{1}$ state, within a time period of $\sim 1$ ps upon the action of pump pulse. On the other hand, from the curve shown in Fig. 11(i) one can see a remarkable gain peak around the 590-nm range produced by the pump pulse. It is a partial evidence of the assumed blueshift effect of gain peak. The reason we did not see a peak shifted to the $\sim 580-\mathrm{nm}$ position is because in our pumpprobe setup, the two-beam's overlapping length is only $\sim 1.5 \mathrm{~mm}$, whereas the whole propagation length for the probe pulse was $\sim 5 \mathrm{~mm}$. Thus the residual linear absorption may partially cover the real gain peak position. 


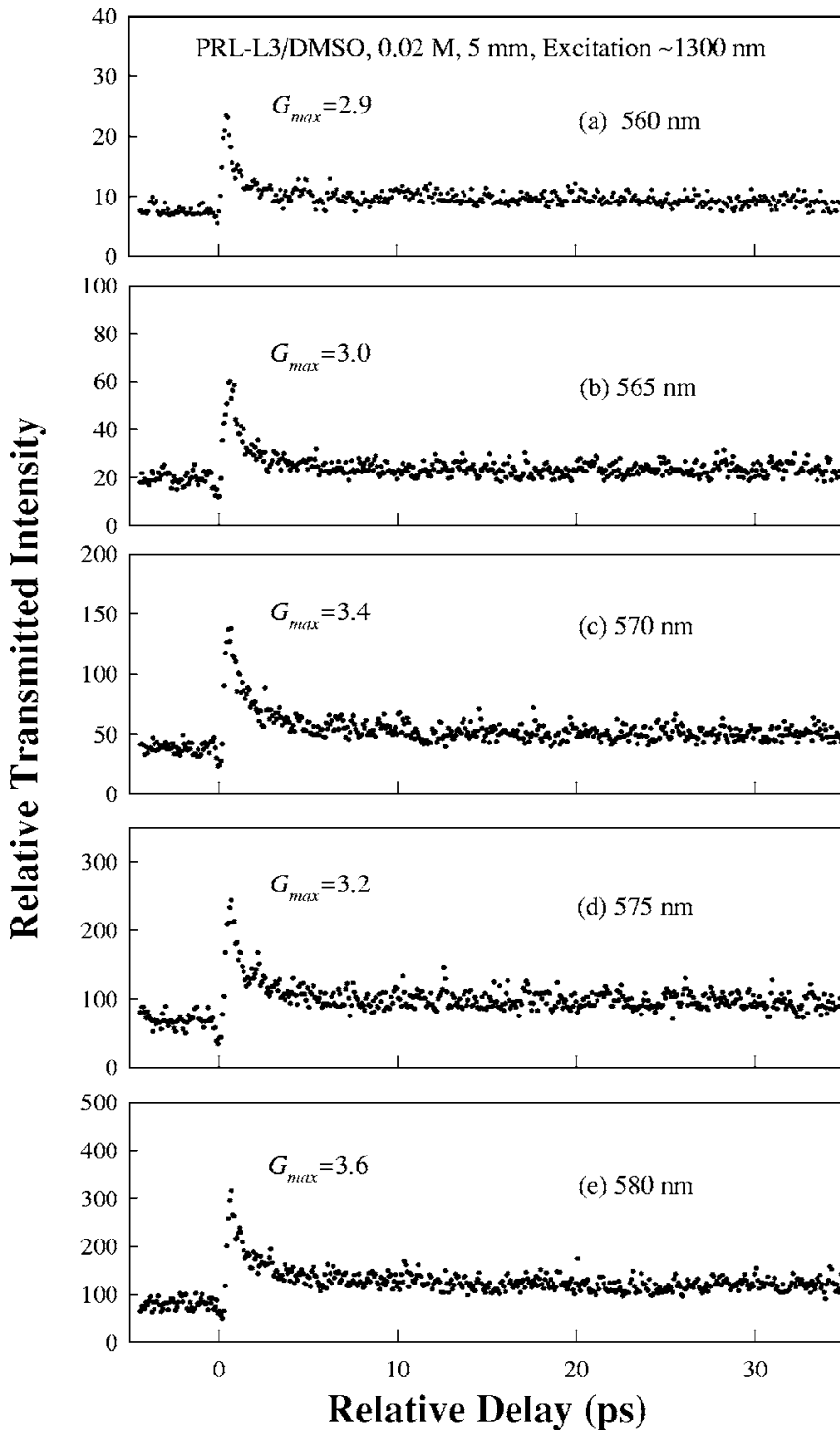

FIG. 12. Temporal profiles of intensity change for five chosen spectral components (from 560 to $580 \mathrm{~nm}$ ) of the transmitted white-light probe beam, indicating the excited-state absorption within a short time period ( $\leqslant 1 \mathrm{ps})$, the transient gain hump, and the long-lasting gain behavior.

To further verify the transient feature of both selfbleaching effect and gain blue-shift effect, the spectrometer shown in Fig. 10 is replaced by a grating monochromator. After passing through this monochromator, different spectral components of the transmitted probe pulse could be detected separately by a photomultiplier tube (PMT) detector connected with a boxcar averager (Model 4420 from EG\&G, Princeton, New Jersey). Thus the temporal intensity change for a chosen spectral component of the transmitted probe pulse can be continuously recorded by smoothly scanning the time delay between the pump and probe pulses. Shown in Fig. 12 are the results of measured temporal behavior of transmitted intensity for five separately chosen spectral components, from 560 to $580 \mathrm{~nm}$ at a 5 -nm wavelength interval, as a function of the relative time delay. Here, each data point is a result of averaging over 50 pulses at $1-\mathrm{kHz}$ repetition,

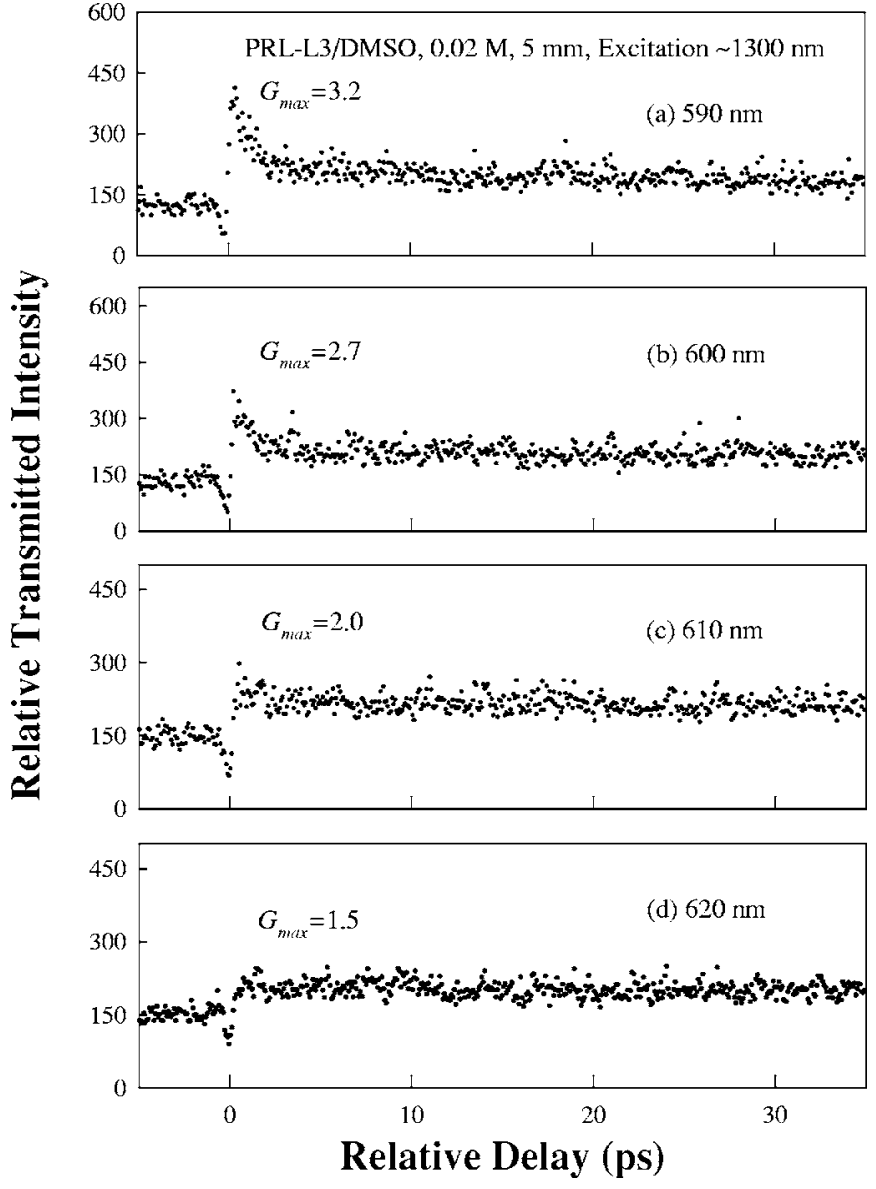

FIG. 13. Temporal profiles of intensity change for four chosen spectral components (from 590 to $620 \mathrm{~nm}$ ) of the transmitted white-light probe beam.

while the time interval between two adjoining points is $79.4 \mathrm{fs}$, which is the temporal resolution of the measured results. From all of the five curves shown in Fig. 12 we can see that at the very beginning there is an excited-stateabsorption induced attenuation that lasts for a time period less than 1 ps that is within the time period of internal conversion and vibrational relaxation we mentioned early. Then there is a fast rising gain peak that lasts more than $3-5 \mathrm{ps}$ in the range of 560-580 nm. Note that the real lasting time of this transient gain may be considerably longer than this apparent value, because the interacting length $(\sim 1.5 \mathrm{~mm})$ between the pump and probe beams is much shorter than the propagation length $(\sim 5 \mathrm{~mm})$ for the probe beam, as we mentioned early. Finally, after the above-mentioned transient gain peak is gone, there still is a very slowly decaying gain, characterizing a steady-state gain behavior. Shown in Fig. 13 are the similar measured results for other four chosen spectral components, from 590 to $620 \mathrm{~nm}$ at a $10-\mathrm{nm}$ interval, obtained under the same conditions. Here, the only difference is that for the spectral components of wavelengths longer than $610 \mathrm{~nm}$, the transient gain behavior is no longer seen, although still there is a long-lasting gain. In Figs. 12 and 13 the measured gain peak values, $G_{\max }=\left(I_{\max }-I_{0}\right) / I_{0}$, of the transient gain hump at different spectral positions are also given, where $I_{0}$ is the intensity of the input signal and 
$I_{\max }$ is the maximum intensity of the transmitted signal. As expected at the $\sim 580-\mathrm{nm}$ position, the measured peak gain value is maximum, which clearly shows the blueshift effect of the transient gain of the dye solution at a high pump level.

The decay of the long lasting steady-state gain approximately follows a single exponential law. The best-fit parameter of the experimental curve shown in Fig. 13(d) is determined to be $\tau=650 \mathrm{ps}$, whereas the measured fluorescence lifetime was $\tau=680 \mathrm{ps}$ in the same sample solution by using a streak-camera method. Based on this comparison, we may conclude that the measured steady-state gain lifetime is nearly the same as the fluorescence lifetime within the experimental uncertainty of $\pm 10 \%$.

\section{CONCLUSIONS}

Under ultrafast three- and four-photon excitation conditions, we have observed the asymmetric properties between the forward and backward stimulated emission pulses. At a high pump level, the peak wavelength of the forward lasing pulse is 20-30 nm shorter than that of the backward lasing pulse for the investigated stilbazolium-salt type of lasing dye solutions. Considering the difference of temporal and spatial sequences for these two oppositely propagating pulse signals, the responses from the excited dye molecules in the effective gain-length range can be essentially different. The forward stimulated emission pulse may experience a transient gain with a peak wavelength located in the blue side of the steady-state fluorescence peak, whereas the backward stimulated emission pulse experiences a steady-state gain profile that is nearly the same as the steady-state fluorescence. The peak wavelength difference of these two types of gain behavior originates from the strong interaction between the salt-type dye molecules and the polar solvent molecules. Combining the transient gain effect and the saturation effect of reabsorption for visible emitted signals, the pump-level dependence of the lasing wavelength shift between the forward and backward pulses can also be well explained. A specially designed two-beam (pump-probe) and time resolved experiment was conducted, in which the probe was a weak ultrashort white-light continuum pulse while the pump was a strong ultrashort $(\sim 160 \mathrm{fs})$ pulse of $\sim 1300 \mathrm{~nm}$ wavelength. The results of this experiment clearly showed the blueshift effect of the transient gain as well as the saturation effect of the reabsorption at the peak wavelength position of the forward lasing pulse.

So far, all experimental results presented in this work are based on three- and four-photon excitation induced lasing processes. However, one may find that the key physical mechanisms proposed here to explain the spectral asymmetry are not dependent on the simultaneously absorbed photon number through which a ground-state dye molecule can be pumped to a higher excited state $S_{i}(i=1$ or 2$)$. In this sense, it can be expected that the similar spectral asymmetry may also be observed in the same type of dye solutions, even with one-photon excitation under appropriate experimental conditions.

\section{ACKNOWLEDGMENTS}

The discussions with Y. R. Shen and H. Ågren are highly appreciated. This work was partially supported by the U. S. Air Force Office of Scientific Research, Washington, D. C. The authors also acknowledge funding from the National Science Foundation, through the international Sweden-US collaboration Program Grant No. DMR-0307282.
[1] G. S. He, J. D. Bhawalkar, C. F. Zhao, C.-K. Park, and P. N. Prasad, Opt. Lett. 20, 2393 (1995).

[2] G. S. He, C. F. Zhao, J. D. Bhawalkar, and P. N. Prasad, Appl. Phys. Lett. 67, 3703 (1995).

[3] G. S. He, L. Yuan, Y. Cui, M. Li, and P. N. Prasad, J. Appl. Phys. 81, 2529 (1996).

[4] G. S. He, K.-S. Kim, L. Yuan, N. Cheng, and P. N. Prasad, Appl. Phys. Lett. 71, 1619 (1997).

[5] A. Abbotto, L. Beverina, R. Bozio, S. Bradamante, C. Ferrante, G. A. Pagani, and R. Signorini, Adv. Mater. (Weinheim, Ger.) 12, 1963 (2000).

[6] Y.-F. Zhou, F.-Q. Meng, X. Zhao, D. Xu, and M.-H. Jiang, J. Phys. Chem. Solids 62, 1145 (2001).

[7] G. S. He, J. D. Bhawalkar, C. Zhao, C. K. Park, and P. N.
Prasad, Appl. Phys. Lett. 68, 3549 (1996).

[8] G. S. He, R. Signorini, and P. N. Prasad, IEEE J. Quantum Electron. 34, 7 (1998).

[9] C. Ye, J. Wang, and D. Lo, Appl. Phys. B 78, 539 (2004).

[10] G. S. He, P. P. Markowicz, T.-C. Lin, and P. N. Prasad, Nature (London) 415, 767 (2002).

[11] G. S. He, J. Dai, T.-C. Lin, P. P. Markowicz, and P. N. Prasad, Opt. Lett. 28, 983 (2003).

[12] G. S. He, R. Helgeson, T.-C. Lin, Q. Zheng, F. Wudl, and P. N. Prasad, IEEE J. Quantum Electron. 39, 1003 (2003).

[13] G. S. He, T.-C. Lin, S.-J. Chung, Q. Zheng, C. Lu, Y. Cui, and P. N. Prasad, J. Opt. Soc. Am. B 22, 2219 (2005).

[14] J. R. Lakowicz, Principles of Fluorescent Spectroscopy (Kluwer Academic/Plenum, New York, 1999), Chaps. 5 and 6. 